\title{
Special training laboratory on optical biophysics
}

\section{Valery Tuchin, Dmitry Zimnyakov, Vladimir Ryabukho, Yurii Sinichkin, Georgy Simonenko, et al.}

Valery V. Tuchin, Dmitry A. Zimnyakov, Vladimir P. Ryabukho, Yurii P. Sinichkin, Georgy V. Simonenko, Ivan V. Fedosov, Alexey N. Bashkatov, Elina A. Genina, Ol'ga A. Perepelitsina, Sergey Ulianov, Ekateryna I. Galanzha, Irina A. Kiseleva, Vyacheslav I. Kochubey, Alexander B. Pravdin, Svetlana P. Chernova, "Special training laboratory on optical biophysics," Proc. SPIE 4588, Seventh International Conference on Education and Training in Optics and Photonics, (28 May 2002); doi: 10.1117/12.468711

SPIE Event: Education and Training in Optics and Photonics 2001, 2001, Singapore, Singapore 
Invited Paper

\title{
Special training laboratory on optical biophysics
}

\author{
Valery V. Tuchin ${ }^{1}$, Dmitry A. Zimnykov, Vladimir P. Ryabukho, Yurii P. Sinichkin, Georgy V. \\ Simonenko, Ivan V. Fedosov, Alexey N. Bashkatov, Elina A. Genina, Ol'ga A. Perepelitsina, \\ Sergey S. Ulianov, Ekaterina I. Galanzha, Irina A. Kiseleva, Vyacheslav I. Kochubey, Alexander B. \\ Pravdin, and Svetlana P. Chernova \\ Saratov State University, Saratov, RU 410026
}

\begin{abstract}
A special training laboratory on optical biophysics for the undergraduate and postgraduate students specialized in biophysics, biochemical physics and medical physics in the framework of subspecialties on biomedical optics, laser medicine, information technologies and mathematical modeling in medicine, and biomedical transducers and sensors is described. The laboratory consists of several sets of topically united practical works: 1) Electronics; 2) Coherent optics of scattering media and interferometry of random phase objects; 3) Coherent-domain optical methods in biomedicine; 4) In vivo reflectance and fluorescence spectroscopy of human skin; 5) Educational-research setups for postgraduate students; 6) Tissue optics and spectroscopy.
\end{abstract}

Keywords: optical biophysics, tissue optics, spectroscopy, scattering media, coherence, curriculum, training laboratory

\section{INTRODUCTION}

Light application in medicine is based on the usage of a great number of phenomena connected with different types of coherent and non-coherent light interaction with tissues and cells. ${ }^{1-5}$ This is the main field of optical biophysics in application for medical science.

One of the most prospective trends of biomedical optics is medical noninvasive optical diagnostics and monitoring of diseases. Light can be absorbed, reflected, scattered and remitted by tissue and each of these processes carries information about the micro- and macrostructure, the movement and shape of its components. The functional imaging and monitoring of the state of human organs and tissues is very attractive feature of the optical method. The complexity of tissue organization and its involvement into living organism (organ) functioning require the development of multidisciplinary approaches for description of tissue optics.

The microscopic, spectroscopic, polarimetric, interferometric, and speckle methods are key techniques for tissue optical imaging and medical diagnosis.

This paper presents curriculum and practical laboratory training for the learning of tissue optics and spectroscopy as a multidisciplinary science by undergraduate and postgraduate students.

\section{CURRICULUM}

Saratov State University educational program on biophysics includes the following specialties: Biophysics, Biochemical physics, and Medical physics with subspecialties: Biomedical optics, Laser medicine, Information technologies and mathematical modeling in medicine, and Biomedical transducers and sensors.

\footnotetext{
1 Department of Physics, Saratov State University, 155, Moskovskaya str., Saratov, 410026, Russia; tel/office: +7
} (8452) 51-46-93, fax: +7 (8452) 24-04-46; e-mail: tuchin@sgu.ru 
Undergraduate training takes 5 academic years. First 3 years students get fundamental knowledge in mathematics, physics, chemistry, biology, biophysics, biochemistry, electronics, informatics, and computing. Humanitarian education is also provided.

This program is developing by Department of Optics of SSU. The following subdivisions are involved in student training: Optics, Radiophysics and nonlinear dynamics, Applied optics and spectroscopy, Applied physics, and Solid state physics.

Disciplines of the specialty are listed in Table 1. It is well seen that Special training laboratory on Optical Biophysics is the most important part of the specialized education, it gives a lot of time for experimental learning of the discipline, three semesters are needed to complete training.

In Table 2 disciplines related to the specialty are listed. These disciples mostly general in physics and chemistry allows for introduction to specialty through a number of biological, physiological or anatomical examples used by lecturers to illustrate physical or chemicals laws and phenomena.

Table 1. Disciplines of the specialty

\begin{tabular}{|l|l|l|l|l|}
\hline$\#$ & Disciplines & Hours & Semester & $\begin{array}{l}\text { Exam/ } \\
\text { Pass-exam }\end{array}$ \\
\hline 1 & Fundamentals of biology (cell biology) & 51 & 4 & Exam \\
\hline 2 & Biochemistry & 72 & 5 & Exam \\
\hline 3 & Biophysics & 51 & 6 & Exam \\
\hline 4 & General training laboratory on Biophysics & 34 & 6 & Pass-exam \\
\hline 5 & Special training laboratory on Optical Biophysics & 322 & $7,8,9$ & Pass-exam \\
\hline 6 & Seminars on annual student project and diploma work & 102 & $2,4,6,8,10$ & Pass-exam \\
\hline
\end{tabular}

Table 2. Disciplines related to the specialty

\begin{tabular}{|l|l|l|l|l|}
\hline$\#$ & Disciplines & Hours & Semester & $\begin{array}{l}\text { Exam/ } \\
\text { Pass-exam }\end{array}$ \\
\hline General & \multicolumn{5}{l|}{} \\
\hline 1 & Chemistry & 36 & 3 & Pass-exam \\
\hline 2 & Optics with biophysical examples & 68 & 4 & Exam \\
\hline 3 & Physical chemistry & 68 & 4 & Exam \\
\hline General alternative & 36 & 7 & Pass-exam \\
\hline 4 & Theoretical optics & 36 & 7 & Pass-exam \\
\hline 5 & Computer programming in biophysics & 36 & 7 & Pass-exam \\
\hline 6 & \begin{tabular}{l} 
Physical basis of mass- and energy-transfer in \\
\multicolumn{2}{|l|}{ biosystems }
\end{tabular} & 34 & 8 & Pass-exam \\
\hline 7 & Fundamentals of photobiology & 34 & 8 & Pass-exam \\
\hline 8 & Electro- and magneto-biology & 34 & 8 & Pass-exam \\
\hline 9 & Quantum biochemistry and molecular structure & 54 & 9 & Pass-exam \\
\hline 10 & Statistical methods in biology and medicine & 54 & 9 & Pass-exam \\
\hline 11 & Deterministic chaos & 54 & 9 & Pass-exam \\
\hline 12 & Fundamentals of opto-acoustic & \multicolumn{3}{l|}{} \\
\hline Optional & 36 & 1 & Review-report \\
\hline 13 & Introduction to specialty & 34 & 8 & Review-report \\
\hline 14 & Safety of life & & \\
\hline
\end{tabular}

Table 3 illustrates the most dynamic set of disciplines - disciplines of subspecialties, some of which can be changed during a few years in accordance with the novel trends in the field. 
Table 3. Disciplines of subspecialties biomedical optics and laser medicine

\begin{tabular}{|l|l|l|l|l|}
\hline$\#$ & Disciplines & Hours & Semester & Exam/Pass-exam \\
\hline 1 & Computer application in research & 68 & 2 & Exam \\
\hline 2 & $\begin{array}{l}\text { Computer-aided systems in optical and biophysical } \\
\text { experiments }\end{array}$ & 51 & 6 & Exam \\
\hline 3 & Physiology of man and animals & 34 & 6 & Pass-exam \\
\hline 4 & Theory of radiation & 36 & 7 & Pass-exam \\
\hline 5 & Lasers and fiber optics for medicine & 36 & 7 & Pass-exam \\
\hline 6 & Coherent-domain methods in optical biophysics & 54 & 7 & Exam \\
\hline 7 & Laser physics and technology & 34 & 8 & Pass-exam \\
\hline 9 & Molecular optics and spectroscopy of biophysics of & 61 & 8 & Exam \\
\hline 10 & $\begin{array}{l}\text { Fundamentals of biochemistry and } \\
\text { photobiological processes }\end{array}$ & 8 & Exam \\
\hline 11 & Nonlinear optics & 51 & 8 & Pass-exam \\
\hline 12 & Analysis of complex biophysical processes & 8 & Exam \\
\hline 13 & Tissue optics & 36 & 9 & Exam \\
\hline 14 & Matrix biomedical optics & 36 & 9 & Exam \\
\hline 15 & Statistical biomedical optics & 36 & 9 & Pass-exam \\
\hline 16 & Luminescence and its biomedical application & 9 & Exam \\
\hline
\end{tabular}

\section{GENERAL DESCRIPTION OF THE TRAINING LABORATORY}

As it is seen from above the special training laboratory on optical biophysics for the undergraduate students specialized in biophysics, biochemical physics and medical physics is a prolongation of a general training laboratory on biophysics and is directed on training of students to modern optical methods of testing and diagnosis in medicine. The laboratory consists of several sets of topically united practical works.

1) Electronics: Analogous/Digital, Digital/Analogous signal converters and digital processing of experimental data.

2) Coherent optics of scattering media and interferometry of random phase objects (Study of tissue thermal denature by use of the quasi-elastic light scattering; Coherent optical analysis of the tissues with fractal-like structure; Diagnostics of macroscopically inhomogeneous non-stationary media using statistical analysis of dynamic speckles; Collimated and focused spatially-modulated laser beam diffraction on a random phase screen; The interferential optical method for measurements of thin layer thickness; Polarization imaging within a multiple scattering medium).

3) Coherent-domain optical methods in biomedicine (Laser analyzer of retinal acuity (Laser retinometer); Visual acuity determination at coherent and incoherent illumination of an object; Speckle microscope for bio-flow measurements).

4) In vivo reflectance and fluorescence spectroscopy of the human skin (Effect of blood and melanin content on skin reflectance spectra; Erythema-melanin-meter; Determination of hemoglobin oxygenation; Effect of blood and melanin content on skin autofluorescence spectra; Evaluation of sunscreen efficiency; Color analysis of reflectance and fluorescence spectra; Skin color imaging; Polarization reflectance spectroscopy; Skin polarization imaging).

5) Educational-research setups for postgraduate students (Laser Doppler velocimeter; Speckle-interferometric instrument for monitoring of capillary bio-flow; Two-wavelength laser scanning microphotometer; Spatial digital color analyzer of biological objects; Spatial-resolved microspectrophotometer for tissue optical properties and geometry studies: CCD tester).

6) Tissue optics and spectroscopy (Two-integrating sphere spectrophotometer for biological fluids and tissues study; Fiber optic diode array spectrophotometer for dynamic measurements; Fibrous tissue optics and matter diffusion phenomena; Fluorescence study of biological tissues).

Indeed, many of these practical works can be used by the postgraduate students, especially when these students were graduated from Biology or Medical departments. As a development of this training laboratory educational-research setups for postgraduate students were designed (Laser Doppler velocimeter; Speckle-interferometric instrument for monitoring of capillary bio-flow; Two-wavelength laser scanning microphotometer; Spatial digital color analyzer of biological objects; Spatial-resolved microspectrophotometer for tissue optical properties and geometry studies: CCD 
tester; Polarization microscopy of biological objects; Fluorescent microscopy of biological objects). Brief description of the some practical works is presented.

\section{Electronics: Analogous/Digital, Digital/Analogous signal converters and digital processing of experimental data.}

The practical works provides: ${ }^{6}$

- Practice on stationary time sequence analysis using standard software (Mathcad, MATLAB and Microcal Origin) and specially designed algorithms for probability density analysis and digital Fourier transform.

- Practice on digital filtering: construction of digital filtering algorithms for recursive and non-recursive filters (second-order and fourth-order low-frequency, high-frequency, band-pass and notch filters).

- Editing of data sequences with anomalous values and artifacts by use of "Tukey 53X" procedure or median filtering based algorithms.

- The study of static and dynamic characteristics of analog-to-digital converter (non-linearity, statistical and spectral characteristics of digital noise, aperture time, jitter).

- The designing of control and data acquisition software for simple computer-aided system of scientific experiment with one input and one output channels.

The practical works will enable students to:

- Understand basic principles of analog and digital signal processing.

- Acquire practical skill in application of the modern analog and digital signal processing systems for research work.

The requirements to a learning person:

- Knowledge of any high-level programming language ( $\mathrm{C}++$, Pascal, Visual Basic).

- Theoretical background in statistics, spectral and correlation analysis and theory of random processes.

- Theoretical background in computer-aided signal processing systems. ${ }^{11}$

Used equipment:

- $\quad$ Personal computer - PC-Pentium-II.

- Multi-functional I/O card - L-154, L-305 or L-1250 type (12-bit 32-channel analog-to-digital converter, 10-bit digital-to-analog converter and 8-bit digital I/O port; L-1250 card has the built-in ADSP signal processor and 256K RAM).

- Generator of test signal - commercially available signal or noise generator.

The following practical works are provided:

- Study of the analog-to-digital signal conversion procedure and analysis of the static and dynamic parameters of 12 bit analog-to-digital converter.

- Practice in applications of 10-bit digital-to-analog converter for controlling peripheral devices (the development of specialized software and its practical approbation for controlling the electrical-mechanical scanning device).

- Digital processing of experimental time sequences (data editing, signal-to-noise ratio improvement, fast Fourier transform and convolution procedures, wavelet transform of non-stationary time sequences with use of the standard functions from MATLAB toolboxes).

- $\quad$ Practice in applications of the LabVIEW software.

Upcoming practical works:

- Practice on non-stationary time sequence analysis with use of Hilbert transform and wavelet transform.

- Designing of control algorithms and programs for various controllable units: step motor, DC motor, piezo-electric transducer, heating element, light-emitting diode.

- Non-standard usage of standard PC peripheral ports (COM, LPT) in computer-aided systems.

- The study of static and dynamic characteristics of CCD-based image-capture systems (non-linearity, offset, "fat" zero, spatial and temporal noise, influence of the finite exposure time on processing of dynamic images). 


\section{Coherent optics of scattering media and interferometry of random phase objects}

\subsection{Study of tissue thermal denature by use of the quasi - elastic light scattering ${ }^{7}$}

Objectives of the work "Study of tissue thermal denature by use of the quasi - elastic light scattering" are the study of the grounds of quasi-elastic light scattering, the single-scattering tissue model, the speckle method as a diagnostic tool, the controlling of tissue structure by thermal denature, the Arrhenius equation, the estimation of the activation energy of albumen denature.

The goal: to estimate the value of activation energy of the tissue thermal denature.

Method: measurement of dependence of correlation time of speckle intensity fluctuations on tissue temperature, use of the single-scattering model, use of Arrhenius equation. Measurement of the correlation time dependence on tissue temperature allows one to evaluate the activation energy of denature.

Student task: To estimate the value of activation energy of the tissue thermal denature from the log dependence of correlation time on tissue temperature (for albumen $-(60 \pm 15) \mathrm{kkal} / \mathrm{mol})$.

\subsection{Coherent optical analysis of the tissues with fractal-like structure ${ }^{7}$}

The practical work "Coherent optical analysis of the tissues with fractal-like structure" enable students to understand basic principles of coherent optical image processing as the basis for morphological study of thin ex-vivo samples of tissues and tissue replicas and to acquire practical experience and skill in design and application of coherent optical systems such as Wiener spectra analyzers.

Equipment needed: Light source - single-mode He-Ne laser ( $5 \mathrm{~mW})$; Fourier-transforming lens - RF-3 type with 350 $\mathrm{mm}$ focal length; Photodetector - photomultiplier tube mounted on computer-controlled step-motor translation stage; Analog-to-digital converter - L-154 type; Computer - Pentium-II.

Student task: to analyze the penetration capacity of various agents such as sunscreens, drugs, etc. and their effect on structure of the skin epidermal layer.

\subsection{Diagnostics of macroscopically inhomogeneous non-stationary media using statistical analysis of dynamic speckles ${ }^{7}$}

Objectives of the work "Diagnostics of macroscopically inhomogeneous non-stationary media using statistical analysis of dynamic speckles" are the study of the basic principles of statistical and correlation analysis, the dynamic speckles, the diagnostics of flows in multiple scattering media, the experience in applications of frame-grabbing systems.

The goal: to study the time-averaged non-stationary dynamic speckles induced by a laminar flow in a scattering slab.

Method: measurement of the time-dependent contrast of dynamic speckles using the time-averaging procedure.

Equipment needed: Light source - single-mode He-Ne laser $(5 \mathrm{~mW})$; Light delivering and light collecting fibers multimode $400 \mu \mathrm{m}$ optical fibers; Phantom object - 5 - mm thick Teflon slab with $1 \mathrm{~mm}$ channel; flowing liquid - $10 \%$ Intralipid or water-milk mixture; CCD camera - Electrim 1000-L; Computer - Pentium-II.

Student tasks: To analyze the influence of image capture parameters (gain, bias, exposure) on the statistical characteristics of recorded dynamic speckle patterns. To study the dependencies of the time-dependent speckle contrast on the position and depth of laminar flow as well as on the flow rate. To estimate the accuracy of the flow localization in the dependence on illumination and detection conditions and scattering geometry.

\subsection{Collimated and focused spatially-modulated laser beam diffraction on a random phase screen ${ }^{7}$}

Objectives of the work "Collimated and focused spatially-modulated laser beam diffraction on a random phase screen" are the study of the random phase screen as a model of scattering media, the diffraction of a laser beam on a random phase screen, the correlation properties of speckle-fields, the interference of partially-developed speckle-fields. 
The goal: to measure the average statistical parameters of a random phase object.

Method: measurement of contrast of average intensity interference fringes formed at diffraction of a spatiallymodulated laser beam (SMLB) on a random phase object; use of the random phase screen model with Gaussian statistics and approximation of non-boundary probing beam. Laser measuring system with collimated SMLB can be used for testing of biological media, for example, for study of dynamic scattering properties of blood suspension. The usage of the focused SMLB allows for determination of statistical parameters of scattering media by measuring of the contrast of average intensity fringes. The focused SMLB at average fringes can be used for retinal acuity estimation of the human eye at cataract (see Practical Work: "Laser analyzer of retinal acuity"). ${ }^{8}$

\subsection{The interferential optical method for measurements of thin layer thickness ${ }^{7}$}

Objectives of the work "The interferential optical method for measurements of thin layer thickness" are the study of the laser interferometry, the speckle-modulation in the scattered light, the correlation properties of speckle-fields, and the interference of developed speckle-fields. ${ }^{7}$

The goal: to measure the thickness of an inhomogeneous transparent layer $h$ at a known index of refraction $n$ or an index of refraction $n$ at known thickness $h$.

Method: measurement of interference fringes spacing in speckle-modulated laser beam reflected by inhomogeneous transparent layer.

\subsection{Polarization imaging within a multiple scattering medium ${ }^{7}$}

The practical work "Polarization imaging within a multiple scattering medium" enable students to obtain knowledge about propagation of a linearly polarized light in a scattering medium, understand influence of absorption and scattering properties of medium on polarization properties of scattered light, understand fundamentals of polarization spectroscopy and imaging, study the model of polarization decay in a scattering medium, study techniques for polarization imaging within scattering media.

Student tasks: Study of influence of absorption and scattering properties of bulk medium on the polarization degree of forward- and backscattered linearly polarized light (Monte Carlo simulation and experiment). Study of influence of absorption and scattering properties of bulk medium on the quality of reconstructed polarization images of objects imbedded in multiple scattering media. Analysis of multilayer scattering structures with use of polarized light. Analysis of absorber location with use of $2 \mathrm{D}$-spectral measurements of polarization parameters of scattering light.

\section{Coherent-domain optical methods in biomedicine}

\subsection{Laser analyzer of retinal acuity (laser retinometer) ${ }^{8}$}

Objectives of the work "Laser analyzer of retinal acuity (laser retinometer)" are the study of the optics and physiology of eye, the laser interferometry, the speckle-modulation in the scattered light.

The goal: to measure the retinal acuity of the human eye using laser interferometer.

Method: Creation on a retina of an eye of the system of rectilinear interference fringes of the various spacing and orientation.

Table 4. Technical characteristics of the retinometer used in he work

\begin{tabular}{|l|c|}
\hline Range of measurement of the retinal acuity & $0.003-1.2$ \\
\hline Field of vision, degrees (not less) & 5.5 \\
\hline Wavelength of radiation, nm & 635 \\
\hline Change of fringe direction, degrees & 135 \\
\hline Measuring time, seconds (no more) & 120 \\
\hline
\end{tabular}




\begin{tabular}{|l|c|}
\hline Laser power, $\mathrm{mW}$ (no more) & 0.1 \\
\hline Time of continuous work, hours (not less) & 8 \\
\hline Dimensions of the optical block (diode laser), $\mathrm{mm}$ & $150 \times 80 \times 75$ \\
\hline
\end{tabular}

3.2 Visual acuity determination at coherent and incoherent illumination of an object ${ }^{8}$

Objectives of the work "Visual acuity determination at coherent and incoherent illumination of an object" are the study of the optics and physiology of eye, the speckle-modulation in the scattered light, the visual acuity.

The goal: to measure the visual acuity at coherent and incoherent illumination of an object.

Method: observation the line pattern or text in coherent and incoherent illumination. Enable students to: learn physiological optics of an eye, understand physical and physiological limits of eye resolution, and apply optical methods of visual acuity estimation. At visual observation of an object at coherent illumination the subjective speckles are formed on the retina. The minimal size of subjective speckles is defined by the eye pupil aperture $\varepsilon_{\perp \min } \approx \lambda_{0} z / n D$, where $D$ is the pupil diameter, $z$ is the distance from the pupil to the retina, $n$ is the refraction index of the vitreous body. For $\lambda_{0}=633 \mathrm{~nm} \rightarrow \varepsilon_{\perp \min } \approx 5 \mu \mathrm{m}$. The average speckle size is about 3-folds bigger $\left\langle\varepsilon_{\perp}\right\rangle=3 \varepsilon_{\perp \text { min }}$. The speckles complicate observation of fine details of the image and therefore reduce resolution of the visual system. Incoherent illumination of the object (screen) is provided by the laser beam transmitted through a movable ground glass with the speed sufficient for the averaging of the subject speckle-pattern. The size of dynamic speckles $\varepsilon_{d}$ on the screen surface should be less, than the diameter $d$ of the resolution area of an eye, $\varepsilon_{d}<d, d \approx \lambda_{0} z_{0} / D, z_{0}$ is the distance from the eye to the screen.

Student task: to determine visual acuity of his/her own eye at coherent and incoherent illumination using the set of scattering screens with black and white parallel strips of various density.

\subsection{Speckle microscope for bio-flow measurements ${ }^{8}$}

Objectives of the work "Speckle microscope for bio-flow measurements" are the study of the measurements of blood and lymph flow velocity in microvessels. In contrast to blood the lymph contains much less of elements, and the cells in the lymph are moving with lower velocity. Lymph flow is characterized by oscillations. Transport of lymph depends mostly on active driving forces caused by lymphatics intrinsic contractility and valve work. Measured parameters: Diameter of lymphatics $(\mu \mathrm{m})$; Number of lymphatics with phasic contractions (\%); Amplituide of phasic contraction (\%); Rate of contractions (per $1 \mathrm{~min}$ ); Number of lymphatics with working valve (\%); Rate of opening of valve (per 1 $\mathrm{min})$; Number of lymphatics with lymph flow (\%); Absolute lymph flow velocity $(\mu \mathrm{m} / \mathrm{s})$.

Students should perform the following tests:

- Summarize principles of optical methods for study of bio-flows: high-resolution speckle interferometry, transmission microscopy, etc.

- Describe optical properties of blood and lymph.

- Determine the lymph and blood flow velocity in microvessels in absolute and relative units on animal model (rat mesentery) using two optical techniques.

- Explain the relation between flow velocity (lymph and blood) in microvessels and other parameters of microcirculation in the living organism (experimental animal).

- Explain mechanisms of microcirculation alteration caused by the vasoactive drugs, chemical and physical agents, various diseases and pathologies.

- Substantiate the application of optical methods for monitoring of blood and lymph flow in norm and for the disease diagnosis.

\section{In vivo reflectance and fluorescence spectroscopy of the human skin}

Reflectance and fluorescence spectroscopy are probably the most developed among the available optical methods for investigating skin in vivo. Reflectance and fluorescence from skin carry information on the structure of epidermis and 
dermis, on the quantity and density of blood vessels, on the concentration and spatial distribution of chromophores and fluorophores in skin, and on the nature of skin metabolic processes.

The latest improvements in fiber optics, electronics, and computer technologies have made reflectance spectroscopy a common and popular technique for analysis of skin. Typical applications include the in vivo quantitative analysis of skin erythema and pigmentation, determination of cutaneous color variation, monitoring of dermatological treatment effects, and study of skin biophysics.

A number of particular applications of fluorescence spectroscopy have already been identified as very useful in the study of skin. These include UVA light excited fluorescence, the use of fluorescence for diagnostic purposes, determination of skin photoaging, determination of the level of primary melanin deposits, assessment of skin erythema and pigmentation, and diagnosis of skin tumors. Methods of skin imaging for histological purposes, based on skin autofluorescence (AF), are also being intensively developed.

The absorption and scattering properties of the skin affect both the AF and the reflectance spectra. Therefore, the combined use of fluorescence and reflectance may provide additional information for the analysis of skin tissue and the study of its biophysical processes.

In this set of practical works the potential advantages and possible uses of the combined use of reflectance and fluorescence spectroscopy of skin for the evaluation of the erythema and pigmentation indices, the determination of the hemoglobin oxygenation and concentration as well as the investigation of the efficacy of topical sunscreens are present. Sometimes, changes in skin reflectance and fluorescence spectra as a result of morphological and functional changes in skin, or as a result of treatment effects, are adequately analyzed with the help of simple models. Such an approach, often leads to new diagnostic methods.

The skin is unique in the sense that it is an organ highly accessible to direct visual inspection. Visual inspection of cutaneous morphology is the mainstay of clinical dermatology. A change of the skin color is one of the most important clues to the severity of the skin lesion and the interpretation of the skin test reaction. Besides color, the exact shape of the lesion play an important role in performing diagnosis. Visualization, documentation, monitoring, measurement, and classification of morphologic manifestations of various cutaneous processes has attracted the use of digital imaging techniques, which are based on computer processing of skin images. Recently, digital color imaging techniques have found various applications in dermatology, such as in melanoma screening, psoriasis and erythema detection, color analysis of nevi, monitoring of wheal and flare reactions, etc.

One example of the examination of skin utilizing imaging techniques is based on analysis of the reflected polarized light. The major advantages in polarization imaging of the skin are that images give the possibility to selectively probe surface or subsurface features of the skin. The quality of the images is such that retrospective evaluation is possible and yields results that compare well with clinical assessment.

The set of practical works includes the following: "Effect of blood and melanin content on skin reflectance spectra", "Erythema-melanin-meter", "Determination of hemoglobin oxygenation", "Effect of blood and melanin content on skin autofluorescence spectra", "Evaluation of sunscreen efficiency", "Color analysis of reflectance and fluorescence spectra", "Skin color imaging", "Polarization reflectance spectroscopy", and "Skin polarization imaging".

These practical works enable students to summarize principles of diffuse reflectance and autofluorescence spectroscopy for in vivo skin examination, to understand fundamentals of polarization spectroscopy and imaging, to explain mechanisms of structural and functional alterations of the human skin caused by skin pathology or external action, and to substantiate the application of the updated optical methods for monitoring of skin structure or functioning.

4.1 "Effect of blood and melanin content on skin reflectance spectra" and "Erythema-melanin-meter" 9 Objectives of the works "Effect of blood and melanin content on skin reflectance spectra" and "Erythema-melaninmeter" are the study of grounds of the human skin diffuse reflectance spectrum forming, the influence of absorption and scattering properties of the human skin on skin diffuse reflectance spectrum, the simple skin model for the analysis of the skin diffuse reflectance spectrum, the quantitative evaluation of blood and melanin content in the human skin with 
use of "pigment indices" (erythema index and melanin index) as well as techniques for determination of erythema and melanin indices. These objectives realize through the following student tasks: the determination of the skin type (I-IV), the determination of the optical density temporal dynamics for skin with developing erythema and for compressed skin, the determination of blood content alteration for skin with developing erythema and for compressed skin, the evaluation of the melanin content in the treated skin areas, and the determination of erythema and melanin indices.

\subsection{Determination of hemoglobin oxygenation ${ }^{9}$}

Objectives of the work "Determination of hemoglobin oxygenation" are the study of techniques for the determination of hemoglobin oxygenation by reflectance spectroscopy and realize through the experimental determination of temporal dynamics of hemoglobin oxygen saturation for the skin of subject at physical training or at vessels occlusion.

\subsection{Effect of blood and melanin content on skin autofluorescence spectra ${ }^{9}$}

Objectives of the work "Effect of blood and melanin content on skin autofluorescence spectra" are the study of grounds of the human skin autofluorescence spectrum forming, the study of skin fluorophores, the simple skin model for the analysis of the skin AF spectrum, and the quantitative evaluation of blood and melanin content. These objectives realize through the following student tasks: the experimental investigation of effective optical density temporal dynamics for skin with developing erythema and for compressed skin, the determination of blood content alteration for skin with developing erythema and for compressed skin, and analysis of influence of a topically applied cream containing melanin on skin AF.

\subsection{Evaluation of sunscreen efficiency ${ }^{9}$}

Objectives of the work "Evaluation of sunscreen efficiency" are the study of effects of topically applied sunscreen creams, techniques for sunscreen testing, the sunscreen transmittance determination using the skin autofluorescence. The student task is the experimental investigation of temporal dynamics of sunscreen protection factor $\mathrm{SPF}(\mathrm{A})$ of commercially available formulations.

\subsection{Color analysis of reflectance and fluorescence spectra ${ }^{9}$}

Objectives of the work "Color analysis of reflectance and fluorescence spectra" are the study of the basis principles and hardware of chromametry, the study of CIE1931 (Yxy) and CIE1976 (L*a*b*) color systems, and the grounds of color perception of skin reflectance and autofluorescence. The student tasks are the investigation of temporal dynamics of reflectance and AF color perception for skin with developing erythema and for compressed skin.

\subsection{Skin color imaging ${ }^{9}$}

Objectives of the work "Skin color imaging" are the study of basic principles and hardware of color imaging, the grounds of skin color a well as techniques for skin color imaging. The student tasks are the assessment of the skin type, the investigation of the skin with erythema, the determination of 2D-distribution of skin erythema and melanin indices.

\subsection{Skin polarization reflectance spectroscopy ${ }^{9}$}

Objectives of the work "Skin polarization reflectance spectroscopy" are the study the propagation of a linearly polarized light in a scattering medium, the formation of co- and cross-polarized components of the back scattered light, and study of techniques for spectral polarization visualization. The student tasks are the investigation of polarization degree and polarization difference spectra for skin with erythema, for compressed skin, for skin at epidermal stripping, as well as for skin treated by a topically applied immersion agent.

\subsection{Skin polarization imaging ${ }^{9}$}

Objectives of the work "Skin polarization imaging" are the study the propagation of a linearly polarized light in a scattering medium, the study of the model of polarization decay in a scattering medium as well as techniques for polarization imaging. The student tasks are the determination of $2 \mathrm{D}$ - distribution of melanin and blood vessels location, the analysis of influence of locally applied melanin contained agents and locally induced erythema on contrast of skin polarization images. 


\section{Educational-research setups for postgraduate students}

The set of practical works includes the following: "Laser Doppler velocimeter"; "Speckle-interferometric instrument for monitoring of capillary bio-flow"; "Two-wavelength laser scanning microphotometer"; "Spatial digital color analyzer of biological objects"; "Spatial-resolved microspectrophotometer for tissue optical properties and geometry studies: CCD tester".

The requirements to a learning person: background in light scattering, tissue optics and spectroscopy.

\subsection{Laser Doppler velocimeter ${ }^{10}$}

This practical work will enable students: to understand principles of optical heterodyning and digital signal processing, to get an overview of principles and schemes of laser instruments such as laser Doppler anemometers (LDA).

Basic kit: laser Doppler velocimeter; rotating screen with motor; set of transparent tubes and scattering fluid.

Examples of practical tasks available with the basic kit:

1. Investigation of physical principles of LDA using rotating scattering screen.

2. Investigation of flow velocity distribution in the tube for Newtonian and non-Newtonian (whole blood) liquids.

3. Development of digital signal processing and analysis for flow dynamics monitoring.

\subsection{Speckle-interferometric instrument for monitoring of capillary bio-flow ${ }^{10}$}

This practical work will enable students: to get an overview of dynamic speckles properties; to identify the time correlation-spectral and space correlation approaches in speckle dynamics analysis; to understand the basic principles of homodyne photodetection and speckle-interferometry.

Examples of tasks for students:

1. Investigation of dynamic speckle field time-dependent intensity fluctuations in a fixed point.

2. Investigation of dynamic speckle field space-time correlation.

3. Monitoring of non-stationary capillary flow.

\subsection{Two-wavelength laser scanning microphotometer ${ }^{10}$}

This practical work will enable students: to get an overview of tissue optical properties; to understand principles of photometry; to analyze spectral images of tissue samples. Examples of tasks for students: investigation of pigment absorption at different wavelengths and concentrations; imaging of tissue sample at two wavelengths; programming of automated sample scanning.

\subsection{Spatial digital color analyzer of biological objects ${ }^{10}$}

This practical work enables students: to obtain knowledge of principle of digital analysis of color image of biological objects; to study technique for measurement of transmittance and reflectance of biological objects using their digital images; to study inverse Monte Carlo method for estimation of tissue optical properties; to estimate optical properties of human hair by inverse Monte Carlo method and spatial digital image analysis.

To evaluate the clinical morphology of different skin lesions (pigmentation, psoriasis, erythema, etc.), measurement of hair growth, wound healing, and burn management digital imaging methods have been applying. Digital imaging techniques combined with inverse Monte Carlo method can be used for estimation of optical properties of human hair shafts.

To obtain optical properties of the human hairs the following steps have to be done: (1) to record images of the hair in both reflectance and transmittance modes using experimental setup; (2) to process obtained images with the developed software allowing for getting of selected red, green, and blue components of the image; (3) to determine reflectance and transmittance of the hair for each color components; (4) to calculate the optical properties of a hair shaft (absorption and reduced scattering coefficients) using experimental data for reflectance and transmittance and inverse Monte Carlo method. 
The color imaging system is composed of a video-microscope (videocamera (SVHS Sony CCD-TR617E, PAL, Japan) and light microscope) interfaced with a personal computer. The specimen (plane plate with attached biological object) is illuminated by white light (halogen lamps provide illumination for recording of transmittance or reflectance images. In dependence on mode of the illumination the specimen plate presents either transparent glass plate (transmittance mode) or black \& white test-object to provide the similar conditions of registration of images (reflectance mode).

Using measured transmittance and reflectance and inverse Monte Carlo method, absorption coefficient $\mu_{\mathrm{a}}$ and reduced scattering coefficient $\mu_{s}^{\prime}$ of the hair shaft at three spectral ranges can be estimated (see Table 5).

Table 5. Optical properties of different hair shafts obtained by inverse Monte Carlo method.

\begin{tabular}{|l|c|c|c|c|c|c|}
\hline \multirow{2}{*}{ Hair color } & \multicolumn{2}{c|}{ Red component } & \multicolumn{2}{c|}{ Green component } & \multicolumn{2}{c|}{ Blue component } \\
\cline { 2 - 7 } & $\mu_{\mathrm{a}}, \mathbf{1 / c m}$ & $\boldsymbol{\mu}_{\mathbf{s}}^{\prime}, \mathbf{1 / c m}$ & $\boldsymbol{\mu}_{\mathrm{a}}, \mathbf{1 / c m}$ & $\boldsymbol{\mu}_{\mathbf{s}}^{\prime}, \mathbf{1 / c m}$ & $\boldsymbol{\mu}_{\mathrm{a}}, \mathbf{1} / \mathbf{c m}$ & $\boldsymbol{\mu}_{\mathbf{s}}^{\prime}, \mathbf{1 / c m}$ \\
\hline Black & $28.5 \pm 6.3$ & $37.5 \pm 18.8$ & $38.6 \pm 7.7$ & $53.4 \pm 35.6$ & $68.3 \pm 8.99$ & $91.3 \pm 91.3$ \\
\hline Brown & $14.5 \pm 3.8$ & $40.6 \pm 17.4$ & $19.71 \pm 5.7$ & $55.8 \pm 29.01$ & $34.82 \pm 8.9$ & $90.5 \pm 48.3$ \\
\hline Light brown & $1.16 \pm 0.064$ & $105.9 \pm 45.1$ & $1.58 \pm 0.061$ & $142.3 \pm 71.3$ & $2.79 \pm 0.13$ & $223.2 \pm 167.3$ \\
\hline Blond & $0.45 \pm 0.074$ & $161.1 \pm 40.3$ & $0.78 \pm 0.12$ & $172.2 \pm 42.5$ & $1.93 \pm 0.28$ & $229.1 \pm 91.6$ \\
\hline Grey & $0.35 \pm 0.054$ & $233.6 \pm 63.6$ & $0.6 \pm 0.1$ & $268.3 \pm 83.01$ & $1.48 \pm 0.2$ & $331.4 \pm 118.2$ \\
\hline
\end{tabular}

The differences between optical properties of various types of hair shafts are well seen. These differences are directly connected with hair shaft structure. High scattering observed for gray hairs is explained by the presence of air-bubbles within the hair shaft. The different content of melanin granules within various shafts (black, brown, etc) causes difference in absorption properties. Melanin granules having higher refractive index than surrounding medium (keratin) also give input in light scattering.

Student tasks:

1. Estimation of transmittance and reflectance of different types of human hair shafts using their digital images;

2. Estimation of absorption and reduced scattering coefficients of the human hair shafts;

3. Analysis of spectral dependence of the absorption and reduced scattering coefficients;

4. Explanation of the differences between optical properties of different types of hair shafts types on the basis of their morphology.

5.5 Spatial-resolved microspectrophotometer for tissue optical properties and geometry studies: CCD tester ${ }^{10}$

The object under study is placed in the object plane of the imaging lens (microscope objective with magnification equal to 8 and numerical aperture equal to 0.20 ) and is illuminated by a fiber-optic illuminator (cross-section diameter is equal to $6 \mathrm{~mm}$ ) assembled with interference filters (bandwidth centered at 600,700 or $800 \mathrm{~nm}$ ). Prism is used to change the optical axis direction. Image of the part of the object is formed on the photosensitive area of the black \& white spectral CCD camera (Electrim 1000). Camera operation is supported by the special software developed by camera producer Electrim Inc. This software allows one to save images of the object under study in 8-bit bitmap format. Saved image can be processed by the special Mathcad (Mathsoft Inc., USA) program allows one to find 2d distributions of the object transmittance for selected wavelength $(600,700$ or $800 \mathrm{~nm})$ and to measure its geometrical parameters. For example, for given number of pixels in rows and columns of CCD chip $(192 \times 165$ for non-interlace mode) the number of pixels in the transverse direction of the hair shaft image is approximately equal to 35 (for $50-\mu \mathrm{m}$ hair diameter). To measure the hair diameter preliminary calibration by using precision $50-\mu \mathrm{m}$ grid on the glass substrate is applied.

Table 2. Measured and calculated transmission $(T)$, absorbance $(A b s)$ and attenuation coefficient $\left(\mu_{4}\right)$ for hair shafts and their mean square deviations (SD) averaged for 27 samples. Attenuation coefficient $\left(\mu_{\mathrm{t}}\right)$ was calculated for diameter $d=50 \mu \mathrm{m}$.

\begin{tabular}{|c|c|c|c|}
\hline Parameter & $\begin{array}{c}\text { CCD, } \\
\lambda=624 \mathrm{~nm}\end{array}$ & $\begin{array}{c}\text { CCD, } \\
\lambda=700 \mathrm{~nm}\end{array}$ & $\begin{array}{c}\text { CCD, } \\
\lambda=800 \mathrm{~nm}\end{array}$ \\
\hline$T, \%$ & $55.9 \pm 8.8$ & $60.6 \pm 6.5$ & $71.6 \pm 10.6$ \\
\hline $\boldsymbol{A b s}$ & $0.61 \pm 0.16$ & $0.50 \pm 0.10$ & $0.33 \pm 0.08$ \\
\hline$\mu_{\mathrm{t}} \mathrm{cm}^{-1}$ & $122 \pm 42$ & $100 \pm 37$ & $66 \pm 25$ \\
\hline
\end{tabular}




\section{Student tasks:}

1. To measure geometric parameters of the human hair shafts.

2. To measure transmission and calculate absorbance and attenuation coefficient of the human hair shafts.

3. To measure erythrocytes size changes at glucose action.

\section{CONCLUSION}

The curriculum and brief description of practical laboratory training on optical biophysics for the experimental learning of tissue optics and spectroscopy as a multidisciplinary science by undergraduate and postgraduate students are presented.

The laboratory consists of several sets of topically united practical works on electronics, coherent optics of scattering media and interferometry of random phase objects, coherent optical methods in biomedicine, tissue optics and spectroscopy, In vivo reflectance and fluorescence spectroscopy of human skin. Educational-research setups for postgraduate students are also briefly described.

More detailed description of many of listed above practical works is presented in the following papers. ${ }^{6-10}$

\section{ACKNOWLEDGMENTS}

Some of presented results were received during researches supported by CRDF grant REC-006 and the Russian Basic Research Foundation grant \#00-15-96667, "Leading Scientific Schools."

\section{REFERENCES}

1. G. Mueller, B. Chance, R. Alfano, S. Arridge, J. Beuthan, E. Gratton, M. Kaschke, B. Masters, S. Svanberg, P. van der Zee (Eds), Medical Optical Tomography: Functional Imaging and Monitoring, SPIE Institutes Series, IS11, 1993.

2. V.V. Tuchin (Ed.), Tissue Optics: Applications in Medical Diagnostics and Therapy, SPIE Milestone Series, MS102, 1994.

3. B.R. Masters (Ed.), Confocal Microscopy, SPIE Milestone Series, MS131, 1996.

4. O. Minet, G. Mueller, and J. Beuthan (Eds.), Selected Papers on Optical Tomography, Fundamentals and Applications in Medicine, SPIE Press, Bellingham, MS147, 1998.

5. V.V. Tuchin, Tissue Optics. Light Scattering Methods and Instrumentation for Medical Diagnosis, SPIE Tutorial Texts in Optical Engineering, TT38, 2000.

6. Dmitry A. Zimnykov and Valery V. Tuchin, "Special training laboratory on optical biophysics. Electronics," Proc SPIE 4707-73, 2002.

7. Dmitry A. Zimnykov, Vladimir P. Ryabukho, Yurii P. Sinichkin, and Valery V. Tuchin, "Special training laboratory on optical biophysics. Coherent optics of scattering media and interferometry of random phase objects," Proc SPIE 4707-74, 2002.

8. Vladimir P. Ryabukho, Sergey S. Ulianov, Ekaterina I. Galanzha, and Valery V. Tuchin, "Special training laboratory on optical biophysics. Coherent-domain methods in biomedicine," Proc SPIE 4707-75, 2002.

9. Yurii P. Sinichkin, Irina A. Kiseleva, Dmitry A. Zimnykov, and Valery V. Tuchin, "Special training laboratory on optical biophysics. In vivo reflectance and fluorescence spectroscopy of the human skin," Proc SPIE 4707-76, 2002.

10. Ivan V. Fedosov, Alexey N. Bashkatov, Elina A. Genina, Georgy V. Simonenko, Dmitry A. Zimnykov, and Valery V. Tuchin, "Special training laboratory on optical biophysics. Education-research setups for postgraduate students," Proc SPIE 4707-77, 2002.

11. George A. Smith, Computer interfacing, Reed Educational \&Professional Publishing Ltd., 2000. 